\title{
Acoustic iridescence
}

\author{
Trevor J. Cox ${ }^{\text {a) }}$ \\ Acoustics Research Centre, University of Salford, Newton Building, Salford, Greater Manchester M5 4WT, \\ United Kingdom
}

(Received 29 July 2010; revised 10 November 2010; accepted 20 November 2010)

\begin{abstract}
An investigation has been undertaken into acoustic iridescence, exploring how a device can be constructed which alter sound waves, in a similar way to structures in nature that act on light to produce optical iridescence. The main construction had many thin perforated sheets spaced half a wavelength apart for a specified design frequency. The sheets create the necessary impedance discontinuities to create backscattered waves, which then interfere to create strongly reflected sound at certain frequencies. Predictions and measurements show a set of harmonics, evenly spaced in frequency, for which sound is reflected strongly. And the frequency of these harmonics increases as the angle of observation gets larger, mimicking the iridescence seen in natural optical systems. Similar to optical systems, the reflections become weaker for oblique angles of reflection. A second construction was briefly examined which exploited a metamaterial made from elements and inclusions which were much smaller than the wavelength. Boundary element method predictions confirmed the potential for creating acoustic iridescence from layers of such a material. @ 2011 Acoustical Society of America.
\end{abstract}

[DOI: 10.1121/1.3531804]

PACS number(s): 43.20.El, 43.20.Fn [ANN]

Pages: $1165-1172$

\section{INTRODUCTION}

Iridescence structures are ones that show "luminous colors that seem to change when seen from different angles." Examples in nature include ${ }^{2}$ the wings of butterflies, peacock feathers, and the scales of beetles and fish. Iridescence is striking not only because the colors change with angle of observation but also because the colors can be among the purest and most brilliant seen. Indeed many iridescent colors cannot be achieved using pigmentation.

Optical iridescence is created through "structural color." On a microscopic scale there are periodic elements whose thicknesses are comparable to the wavelength of light. Figure 1 shows an image of a butterfly wing magnified using transmission electron microscopy (TEM). ${ }^{3}$ In this case the butterfly is Morpho rhetenor. The periodic elements shown in the image consist of stacks of alternating thin elements. As light propagates through this structure, backscattering occurs at the interface between the thin elements because of the contrasting refractive index of adjacent layers.

If the structure is illuminated by light whose wavelength is four times the thickness of each layer, then an observer normal to the wing will see a bright reflection because the backscattered waves arrive in phase. When observed at an oblique angle, this constructive interference occurs for light of a shorter wavelength and hence the color of the surface changes with angle of observation. For instance, for the butterfly Morpho menelaus the color goes from blue at normal reflection to violet at oblique angles.

The study reported in this paper explored how to construct an acoustic equivalent of this optical iridescent structure. In other words, one where the frequency of reflected

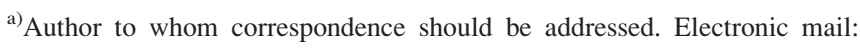
t.j.cox@salford.ac.uk
}

sound increases with angle of reflection. And then to examine the properties that such structures might have.

\section{CONSTRUCTION}

Creating an exact acoustic equivalent of the optical system is not straightforward. The refractive index of the layers found in natural optical systems is relatively close to 1 . In Morpho menelaus, ${ }^{4}$ the thin elements are formed from cuticle and air. The cuticle layers have a refractive index in the range 1.4-1.9, and the air layer has a refractive index of about 1-1.1 (fine filaments of cuticle are present in the air layer which means the refractive index is slightly above 1).

If an exact acoustic analog was to be made, a material with a speed of sound about 1.6 times larger (or smaller) than that in air is needed. In other words, materials where the speed of sound is around 550 or $210 \mathrm{~ms}^{-1}$. In theory this could be achieved using layers of different gases, but then it would be difficult if is not impossible to constrain the gas layers into thin films. A conventional porous absorber such as mineral wool or foam could be used to obtain a lower speed of sound, but this would also cause unwanted absorption.

Sonic (or phononic) crystals ${ }^{5}$ have been much studied in recent decades and have potential for iridescence because they contain periodicity with repeat distances of the same order as a wavelength. Sonic crystals are formed using a periodic array of scattering elements: spheres or cylinders are commonly used. The periodicity prevents some frequencies from being transmitted through the array-these are the bandgaps. Because sonic crystals are periodic, they also reflect sound strongly at certain frequencies, and as the angle of observation changes, the frequencies at which strong reflections occur alters. However, because common sonic crystals are periodic in two or more dimensions, with the geometry in each of the dimensions having size comparable to the wavelength, the way that the frequencies of strong reflection alter 


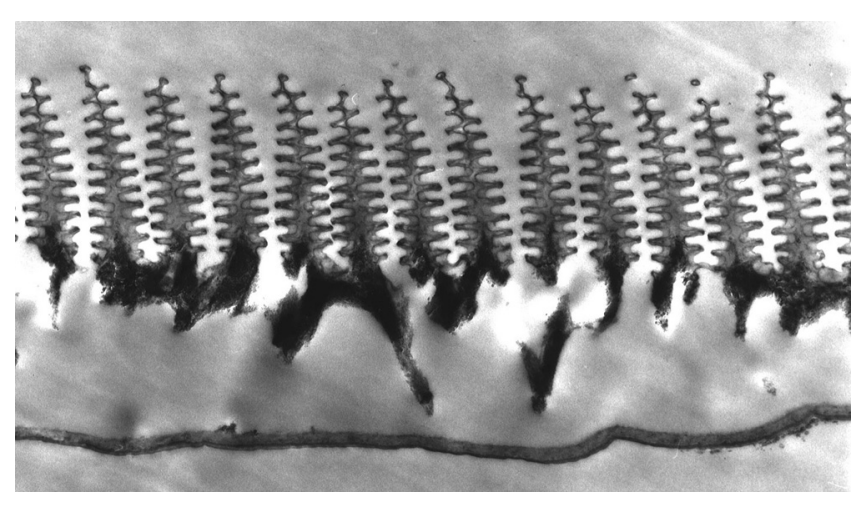

FIG. 1. TEM image from the Morpho rhetenor butterfly (after Vukusic et al. ${ }^{2}$ ) The width of the whole image from left to right is approximately $10 \mu \mathrm{m}$.

with the angle of observation is different to the optical case being mimicked. While some frequencies of strong reflection increase as the angle of observation gets larger, others decrease.

However, some researchers have investigated using anisotropic elements, such as squares or ellipses, on an isotropic grid. The work on elliptical cylinders by $\mathrm{Wu}$ and $\mathrm{Chen}^{6}$ is most relevant to acoustic iridescence. $\mathrm{Wu}$ and Chen used simulation to show that the angle of the transmitted acoustic wave is frequency dependent and can be altered by rotating
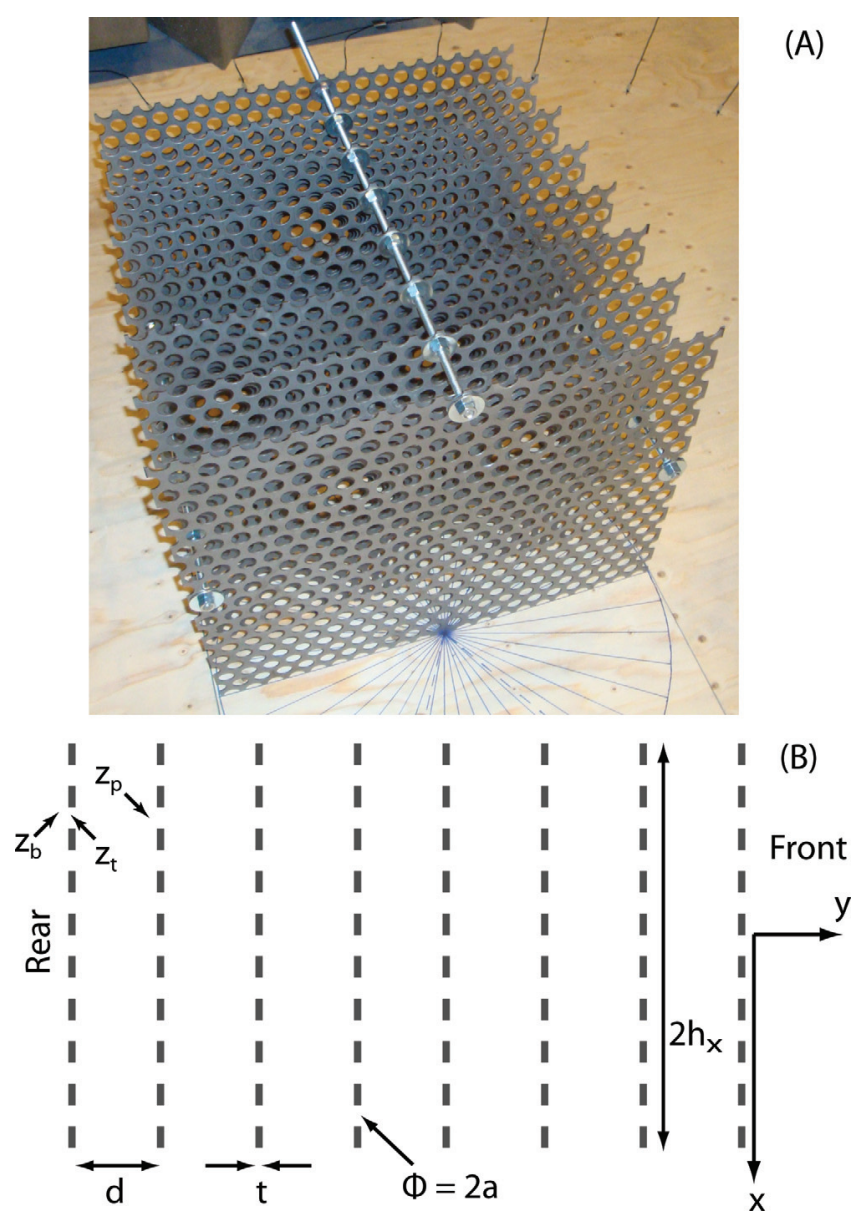

FIG. 2. (Color online) One of the samples tested shown as: (A) a photo and (B) in cross section. In the cross section, the source is to the right. The locations of where the impedances $z_{b}, z_{p}$, and $z_{t}$ are calculated are also indicated. the ellipses within the sonic crystal. The research presented below adds to this work by examining and designing devices from the perspective of acoustic iridescence in the backscattered region. The work below also provides the first experiment evidence of acoustic iridescence.

Consequently, the acoustic analog of structural color requires periodicity only in one dimension. One approach is shown in Fig. 2. In optics, iridescence is caused by backscattering at impedance discontinuities spaced at quarter of a wavelength apart. A similar effect can be achieved in acoustics by placing a series of parallel perforated sheets with half a wavelength between them $\left(d=\lambda_{0} / 2\right.$, where $d$ is the sheet spacing and $\lambda_{0}$ the design wavelength). The perforations are made large enough to minimize the loses due to viscous effects in the holes. Consequently, it is the radiation reactance of the sheets, the mass loading caused by the air vibrating in the perforations, which generate the necessary impedance discontinuities. The mass loading will cause the first strongly reflected frequency to be slightly lower than a simple calculation based on $d=\lambda_{0} / 2$.

\section{NUMERICAL MODELING}

Initial studies were carried out using two modeling techniques. A simplified model based on the well-established transfer matrix approach was used to initially develop possible structures. The most promising devices were then measured. After reviewing the measurement results, it was found necessary to use a more exact boundary element method (BEM) to predict the surface reflection.

\section{A. Boundary element method}

Predictions are based on the Helmholtz-Kirchhoff integral equation. This formulates the pressure at a point, as a combination of the pressure direct from the sources, and a surface integral of the pressure and its derivative over the reflecting surfaces. The single frequency form of the integral equation gives the pressure $p$ as ${ }^{7}$

$$
\begin{aligned}
& \left.\begin{array}{cc}
\mathbf{r} \in E & p(\mathbf{r}) \\
\mathbf{r} \in S & \frac{1}{2} p(\mathbf{r}) \\
\mathbf{r} \in D & 0
\end{array}\right\}=p_{i}\left(\mathbf{r}, \mathbf{r}_{0}\right)+\int_{s} p\left(\mathbf{r}_{s}\right) \frac{\partial G\left(\mathbf{r}, \mathbf{r}_{s}\right)}{\partial n\left(\mathbf{r}_{s}\right)} \\
& -G\left(\mathbf{r}, \mathbf{r}_{s}\right) \frac{\partial p\left(\mathbf{r}_{s}\right)}{\partial n\left(\mathbf{r}_{s}\right)} d s,
\end{aligned}
$$

where $\boldsymbol{r}=\{x, y, z\}$ is the vector describing the receiver location; $\boldsymbol{r}_{0}=\left\{x_{0}, y_{0}, z_{0}\right\}$ is the vector describing the source location; $\boldsymbol{r}_{s}=\left\{x_{s}, y_{s}, z_{s}\right\}$ is the vector for a point on the surface; $p_{i}\left(\boldsymbol{r}, \boldsymbol{r}_{0}\right)$ is the direct pressure radiated from the source at $\boldsymbol{r}_{0}$ to the receiver at $\boldsymbol{r} ; G$ is the Green's function; $\boldsymbol{n}$ is the normal to the surface pointing out of the surface; $E$ is the external region; $s$ is the surface; and $D$ is the interior of the surface (see Fig. 3).

In three dimension (3D) the Green's function is given by

$$
G\left(\mathbf{r}, \mathbf{r}_{0}\right)=\frac{e^{-i k\left|r-r_{0}\right|}}{4 \pi\left|r-r_{0}\right|}
$$




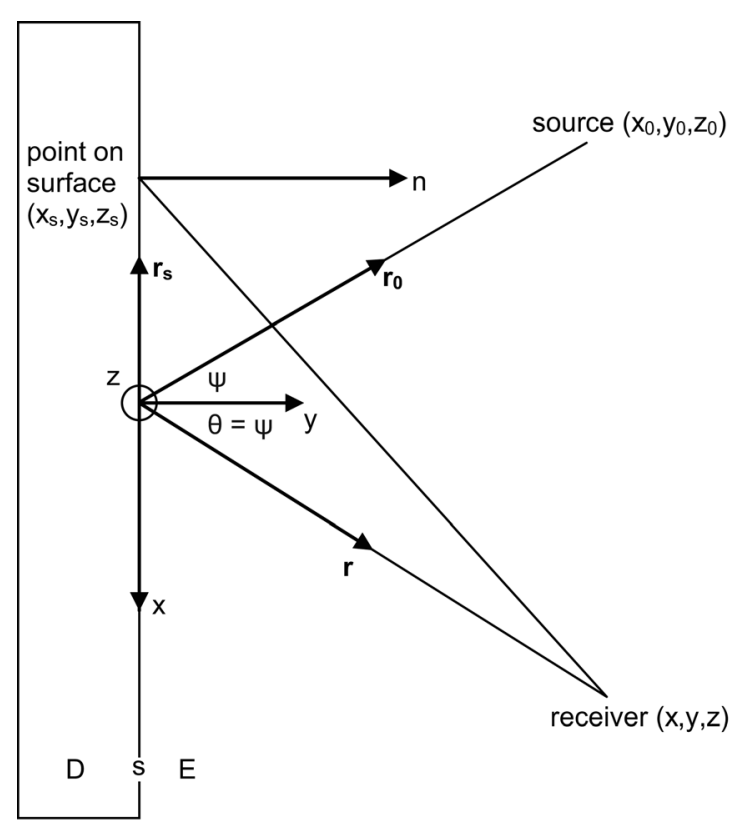

FIG. 3. Configuration for prediction models.

and in two dimension (2D)

$$
G\left(\mathbf{r}, \mathbf{r}_{0}\right)=\frac{-i}{4} H_{0}^{(1)}\left(k\left|r-r_{0}\right|\right)
$$

where $H_{0}{ }^{(1)}$ is the Hankel function of the first kind of order zero.

The most precise method uses a BEM constructed from Eq. (1) and its derivative. As the metal sheets are thin, a formulation in terms of the pressure difference across solid parts of the metal sheets is used. Terai ${ }^{8}$ showed that for points on the surface

$0=\frac{\partial p_{i}\left(\mathbf{r}_{0}, \mathbf{r}_{s, 1}\right)}{\partial n\left(\mathbf{r}_{s, 1}\right)}+\int_{s}\left(p\left(\mathbf{r}_{s, 1}\right)-p\left(\mathbf{r}_{s, 2}\right)\right) \frac{\partial^{2} G\left(\mathbf{r}, \mathbf{r}_{s, 1}\right)}{\partial n\left(\mathbf{r}_{1}\right) \partial n\left(\mathbf{r}_{s, 1}\right)} d s$,

where it is assumed that the sheets are non-absorbing. The subscripts 1 and 2 refer to the front and the back of each infinitesimally thick panel, respectively.

Using Eq. (4), it is possible to discretize the metal sheets into a set of elements across which the pressure is assumed constant, and set up simultaneous equations in terms of the pressure difference between the front and rear of the panel $p\left(\mathbf{r}_{\mathrm{s}, 1}\right)-p\left(\mathbf{r}_{\mathrm{s}, 2}\right)$. Once the pressure difference for each element has been evaluated, then the following formulation is used to calculate the scattered pressure $p_{s}(\boldsymbol{r})$ at receiver positions

$$
p_{s}(\mathbf{r})=\int_{s}\left(p\left(\mathbf{r}_{s, 1}\right)-p\left(\mathbf{r}_{s, 2}\right)\right) \frac{\partial G\left(\mathbf{r}, \mathbf{r}_{s, 1}\right)}{\partial n\left(\mathbf{r}_{s, 1}\right)} d s .
$$

\section{B. Transfer matrix}

Initially, a simpler model was used to explore the surface properties. The transfer matrix approach is well established for predicting propagation within multi-layers of acoustic media, especially porous absorbers. It enables the surface impedance of layered acoustic materials to be calculated and can be applied to the iridescent structures.

It is assumed that only plane waves exist between the perforated sheets. To simplify the formulations, the propagation is constrained to be entirely within the $x-y$ plane (Fig. 2 ). By considering the continuity of pressure and velocity at the boundaries between the perforated sheets and air, it is possible to produce formulations for the impedance at different places within the structure.

The backing impedance for the perforated sheet furthest from the source is assumed to be the characteristic impedance of air, i.e., $z_{b}=\rho c$, where $\rho$ is the density and $c$ is the speed of sound in air (see Fig. 2 for location of impedances).

The impedance on the other side of this perforated sheet $z_{t}$ is then given by ${ }^{9}$

$$
z_{t}=\frac{\rho}{\varepsilon}\left(\frac{t}{2 a}+1\right) \sqrt{8 v \omega}+(2 \delta a+t) \frac{i \omega \rho}{\varepsilon}+z_{b},
$$

$v=15 \times 10^{-6} \mathrm{~m}^{2} \mathrm{~s}^{-1}$ is the kinemetric viscosity of air, $\delta$ is the end correction factor, $\varepsilon$ is the fraction of open area of the perforated sheet, $t$ is the sheet thickness, $a$ is the hole radius, and $\omega$ is the angular frequency. The first term represents the resistance of the sheet, which for the geometries used here is small and can be neglected.

The following formulation is used to calculate the end correction factor (because it allows for very open perforated sheets): ${ }^{10}$

$$
\delta=0.8\left(1-1.47 \varepsilon^{1 / 2}+0.47 \varepsilon^{3 / 2}\right) .
$$

The surface impedance at the top of the air layer, just below the next perforated sheet, $z_{p}$ is given by ${ }^{11}$

$$
z_{p}=\frac{-i z_{t} \rho c\left(k / k_{y}\right) \cot \left(k_{y} d_{i}\right)+\left(\rho c k / k_{y}\right)^{2}}{z_{t}-i \rho c\left(k / k_{y}\right) \cot \left(k_{y} d_{i}\right)}
$$

where $k$ is the wavenumber in air and $k_{y}=k \cos (\psi)$ is the component of the wavenumber in the $y$ direction, where $\psi$ is the angle of incidence. Equations (6) and (8) can then be applied recursively to the whole structure, taking one layer at a time, until the surface impedance at the face of the perforated sheet nearest the source is obtained.

Once this surface impedance is known, this is used to predict the scattered pressure. This is done using a simplified prediction model based on Eq. (1). The surface impedance at the surface of the final sheet allows the pressure and its derivative on the surface to be related in terms of surface admittance, $\beta^{\prime}$

$$
i k p\left(\mathbf{r}_{s}\right) \beta^{\prime}\left(\mathbf{r}_{s}\right)=\frac{\partial p\left(\mathbf{r}_{s}\right)}{\partial n\left(\mathbf{r}_{s}\right)}
$$

where the prime indicates that the admittance is calculated with the velocity pointing out of the surface as is common for BEM implementations. (Note previous formulations for impedance produce the inward pointing value.)

To derive a quick and simple-to-calculate model the Kirchhoff boundary conditions are used ${ }^{12}$ which relates the 
surface pressures on the front sheet to the incidence pressure via the plane wave reflection coefficient $R$

$$
p\left(\mathbf{r}_{s}\right)=(1+R) p_{i}\left(\mathbf{r}_{s}, \mathbf{r}_{0}\right) .
$$

These pressures are taken on the front of the final sheet only (denoted $s_{f}$ in subsequent equations) as the surface impedance formulations include the propagation through the structure to subsequent layers.

Assuming far field scattering allows a simplification of the derivative of the Green's function

$$
\frac{\partial G\left(\mathbf{r}, \mathbf{r}_{s}\right)}{\partial n\left(\mathbf{r}_{s}\right)} \approx-i k G\left(\mathbf{r}, \mathbf{r}_{s}\right) \cos (\theta),
$$

where $\theta$ is the angle of reflection. Equations (9)-(11) are substituted into Eq. (1). For iridescence the specular reflection direction is of most interest when the angle of incidence and reflection are identical. This allows a simplification of the formulations after some manipulation

$$
p_{s}(\mathbf{r})=-2 i k R \int_{S_{f}} p_{i}\left(\mathbf{r}_{s}, \mathbf{r}_{0}\right) G\left(\mathbf{r}, \mathbf{r}_{s}\right) \cos (\theta) d s,
$$

which is a simple numerical integration over the surface and rapid to solve. As this is a far field problem, the formulation can be further simplified using approaches commonly used in optics for Fraunhoffer diffraction ${ }^{13}$

$$
\left|p_{s}(\mathbf{r})\right| \approx-\frac{i k h_{z} h_{x} \cos (\theta) R}{2 \pi^{2} r r_{0}},
$$

where $2 h_{x}$ and $2 h_{z}$ are the width and length of the surface in the $x$ and $z$ directions, respectively.

\section{Two dimensional model}

For the BEM, it was decided to use a 2D model to predict the scattering, because the number of elements required for $3 \mathrm{D}$ predictions was rather large. Using a $2 \mathrm{D}$ model added one additional complication because without care the radiation impedance generated within the $2 \mathrm{D}$ model would not match the measurements. Using the hole diameter and open area from the real perforated sheets to form a 2D BEM mesh with slot width equal to the hole diameter yields inaccurate results.

To overcome this problem, the hole size in the 2D mesh was decreased until the perforated sheet reactance in the 2D BEM matched that expected from the real-world 3D sheet. The expected 3D mesh reactance was calculated using Eqs. (6) and (7). The reactance of a 2D sheet was calculated by simulating a two-microphone free field measurement of surface impedance ${ }^{14}$ using the BEM model. A single perforated sheet with an air-backing was simulated across the frequency range of interested. The size of the holes and open area in the $2 \mathrm{D}$ BEM mesh was then adjusted in a trial-and-error process until the 2D surface reactance better matched the realworld 3D case. This was found to happen when the openings were $1 \mathrm{~cm}$ wide with a $71 \%$ open area.

\section{DESIGN SIMULATIONS}

Before measurement, the transfer matrix method was used to examine the behavior of candidate iridescent devi-

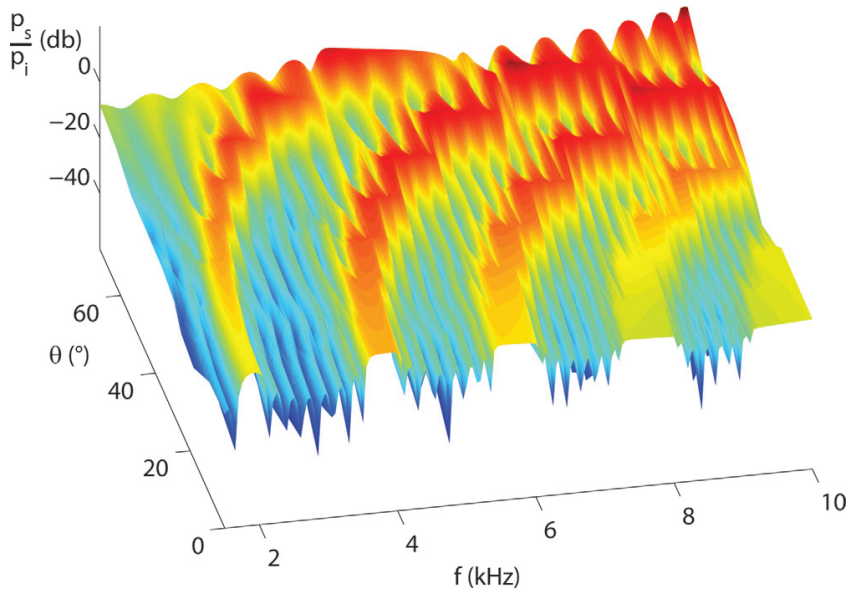

FIG. 4. (Color online) Predicted scattered pressure level as a function of angle and frequency for an iridescent structure with spaced identical perforated plates. Transfer matrix model.

ces. For the first results shown here, the following geometry was used: Sheet thickness, $t=2 \mathrm{~mm}$; open area $\varepsilon=0.46$; sheet spacing $d=8.6 \mathrm{~cm}$; and hole radius $a=1 \mathrm{~cm}$. This gives a nominal design frequency of $1930 \mathrm{~Hz}$.

The source was placed at a radius of $2.5 \mathrm{~m}$ and the receiver at a distance of $1.35 \mathrm{~m}$. These distances were chosen because they were the distances to be used in the measurements. The source and receiver were set at the same angle either side of the normal to the surface so the specular reflection was measured. The sound pressure level shown in the graphs is a ratio of the pressure scattered from the surface referenced to the direct incident sound $p_{i}\left(\boldsymbol{r}, \boldsymbol{r}_{0}\right)$ at the same point.

Figure 4 shows the predicted scattered sound pressure level as a function of frequency and incident/reflection angle. The prediction uses the transfer matrix model. A series of harmonic peaks are seen, and as the angle of reflection gets larger the frequency of each harmonic increases. This is acoustic iridescence mimicking the optical systems. The width of the peaks in the spectra increase as the angle of reflection gets larger.

Unlike optical iridescence, multiple acoustic frequencies are reflected strongly within the bandwidth of interest. This arises because the sound analysis covers many octaves allowing higher order harmonics to be seen. In contrast, the visible optical spectrum has a much narrower bandwidth: as it is only about an octave in extent, higher harmonics are invisible to the human eye.

The sheet impedance includes the end correction due to the radiation impedance of the perforated sheet-something unique to acoustics and without an equivalent in optic structural color. For this reason, the first harmonic peak is at a somewhat lower frequency than the design specification, because of the added mass of the vibrating air in the perforated sheets.

The configuration used in this simulation clearly demonstrates iridescence. The hole size, open area, and sheet thickness were worked out using a numerical optimization. A computer was tasked with searching possible geometries to find the one which gave the clearest and most distinct first harmonics. A structure where the first harmonic had the largest Q-factor for normal incidence was chosen. This was done 


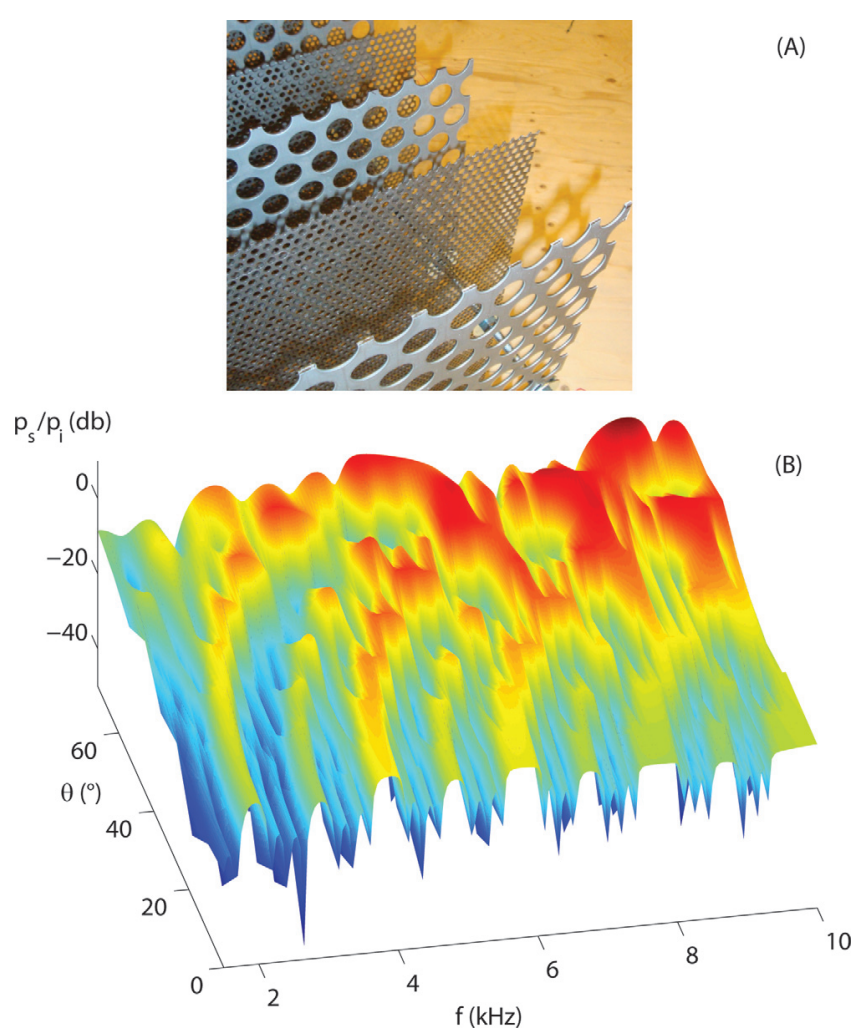

FIG. 5. (Color online) (A) An iridescent structure with two types of perforated plates. (B) Predicted scattered pressure level as a function of angle and frequency for the structure using transfer matrix model.

prior to measurements to try and discover configurations where iridescence was clearest and therefore most likely to be successfully measured.

Initial optimizations had the structures made up from identical perforated sheets for all layers. Further numerical optimizations were carried out where each sheet could be different and the result of design work is shown at the top of Fig. 5. The predicted scattering from the structure is also shown in the figure. The peaks in the spectra have a higher Q-value than shown previously, and there are twice as many of them. The optimization process made alternating sheets have very different hole sizes and spacings, and consequently very different radiation impedances. (In the predictions alternative layers of two sheet configurations were used. The first sheet was as used before to generate Fig. 4, and the other sheet had $t=1 \mathrm{~mm}, \varepsilon=0.46$, and $a=2.5 \mathrm{~mm}$.) Using alternating sheets with different radiation impedances generates an additional low frequency harmonic peak relating to twice the sheet spacing which narrows the peak around the design frequency of $2 \mathrm{kHz}$. Such a construction is not seen in optical systems showing structural color.

\section{MEASUREMENTS}

The two structures which were discussed in Sec. IV were constructed and measured. Figure 2 and the inset of Fig. 5 show photos of the constructions. The perforated sheets were held apart at the correct spacings using nuts and threaded bar. The width $(588 \mathrm{~mm})$ and height $(480 \mathrm{~mm}$ small holes; $485 \mathrm{~mm}$ large holes) of the sheets were chosen to be

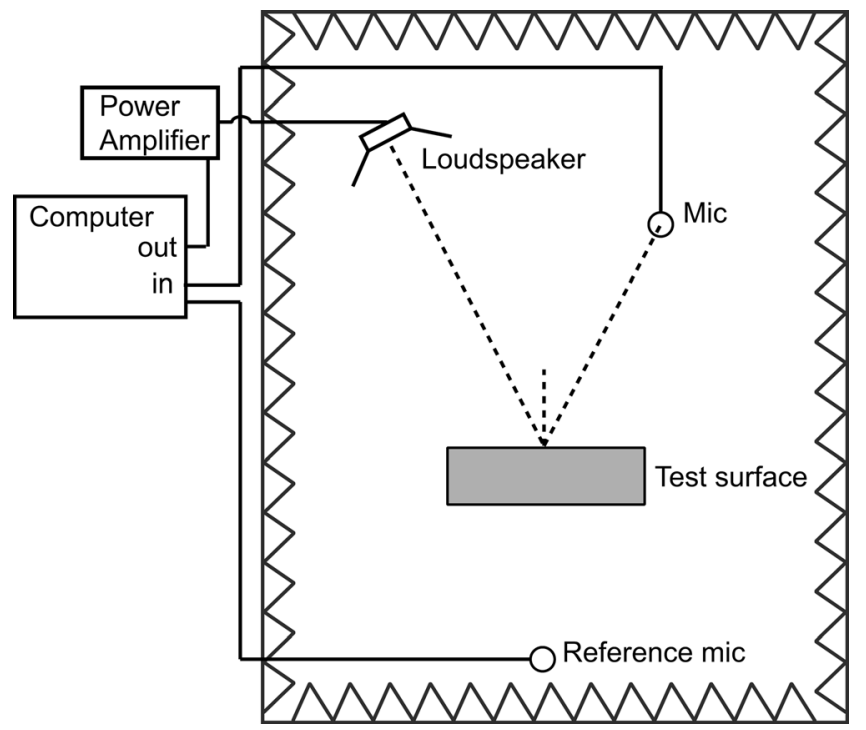

FIG. 6. Plan view of set up for measurement.

at least a couple of wavelengths in extent for the lowest frequency of interest. This was done to minimize the effect that edge diffraction might have on the measured results.

Figure 6 shows a plan view of the measurement set-up used. The procedure was similar to that used to characterize reflections from diffusers, ${ }^{15}$ and so the following description is brief. A boundary layer technique is used where the microphones, loudspeaker, and sample are placed on the floor of a semi-anechoic chamber. This is done for convenience. The floor acts as a mirror image and what is measured is effectively the structure paired with a mirror image of itself reflected in the floor.

A loudspeaker placed on the floor is used to irradiate the test surface with a maximum length sequence at the desired angle of incidence $\psi$. A microphone (on the floor) in the specular reflection direction $(\theta=\psi)$ records the pressure. The impulse response is then recovered via a deconvolution. A time window is applied to the impulse response to isolate just the reflected sound, removing the sound propagating directly from the loudspeaker to the microphone. A Fourier transform is applied to the windowed impulse response to obtain the scattered pressure spectrum. This spectrum is normalized to a measurement of the incident pressure at a reference microphone behind the sample; the reference measurement is taken with no sample present. This normalization removes the frequency responses of the transducers from the results. A measurement was made every $9^{\circ}$ up to an angle of $72^{\circ}$ relative to the surface normal.

\section{RESULTS}

Figure 7 compares the measured scattered pressure level to predictions from the BEM and transfer matrix model. Each graph shows a spectrum for a different angle of incidence/reflection. The results shown are for the structure where all perforated sheets are identical.

The measured spectra display the characteristics of iridescence with the frequency of the harmonics in the scattered pressure spectra increasing as the angle of reflection 

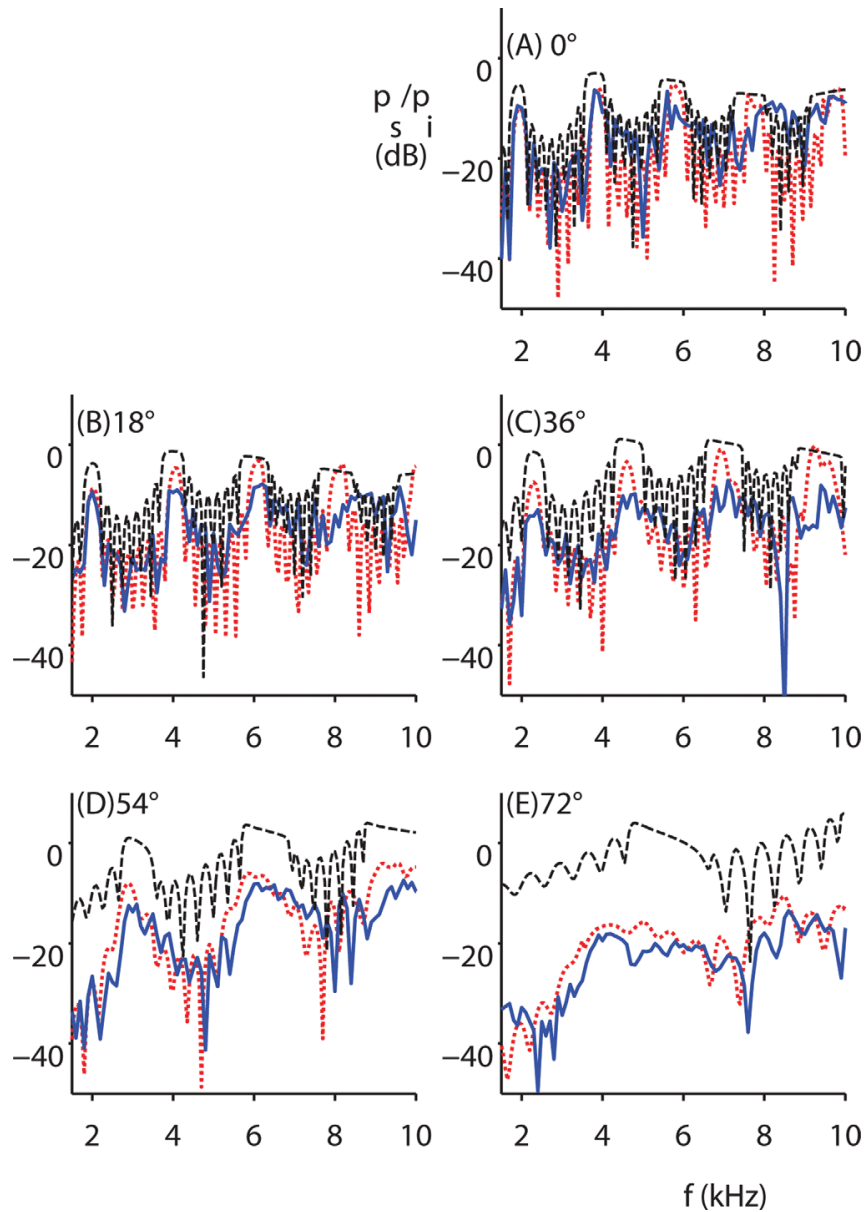

FIG. 7. (Color online) Comparison of scattered pressure level for measurement; -....... BEM prediction and - - - - transfer matrix prediction. Each graph represents a different observation angle: (A) $0^{\circ}$, (B) $18^{\circ}$, (C) $36^{\circ}$, (D) $54^{\circ}$, and (E) $72^{\circ}$. Sample had eight layers. Dimensions of perforated sheets used in measurement $t=2 \mathrm{~mm}, \varepsilon=0.46, \quad a=1 \mathrm{~cm}$, width $=0.588 \mathrm{~m}$, and height $=0.485 \mathrm{~m}$.

gets larger. Although at oblique angles, the measured reflected energy is significantly lower and the harmonics of iridescence become broader and harder to identify.

The transfer matrix model consistently overestimates the scattered pressure level, by an average of $4 \mathrm{~dB}$ for $0^{\circ}$, rising to $20 \mathrm{~dB}$ for $72^{\circ}$. Despite this, it does successfully predict the frequencies of the harmonics. The transfer matrix formulation for the air layers, Eq. (8), properly allows for oblique sound propagation. However, the impedance of the perforated sheets, shown within Eq. 6, becomes more inaccurate as the angle of reflection increases due to increased mutual interactions between the perforations. Eventually, as the angle of reflection approaches grazing, the propagation is parallel to the perforated sheets, and the transfer matrix model completely breaks down.

In contrast, the BEM predictions are much more accurate. The average error between the measured spectra and the $\mathrm{BEM}$ predictions is $3 \mathrm{~dB}$ or less for all angles, and the harmonic frequencies are correctly predicted for all angles of reflection. This happens because the BEM model is a more exact solution of the Helmholtz-Kirchhoff equation, for instance, it does not make assumptions about the radiation impedance.
As the angle of reflection increases, the peaks indicating strong reflection become broader and harder to distinguish. Similar effects are seen in optical systems. ${ }^{3}$ Measurements on Morpho rhetenor indicate that the blue reflection contains $70 \%$ of the incident energy and extends over $\pm 50^{\circ}$. Coincidentally, this is a similar angular range seen in the acoustic measurement which has clear harmonics for $\pm 63^{\circ}$. The intensity of the reflection in the acoustic case is much lower than seen in the Morpho rhetenor butterfly. Between $0^{\circ}$ and $63^{\circ}$, the first harmonic in the acoustic spectra is attenuated by $8.2 \mathrm{~dB}$ when compared to the energy scattered from a non-absorbing box of the same size as the iridescent structure. $(8.2 \mathrm{~dB}$ is equivalent to an intensity of only $15 \%$ ). This can partly be explained by the smaller number of layers in the acoustic structure. However, to significantly increase the reflected sound pressure level requires the geometry to be changed to achieve a stronger backscattering from each perforated sheet.

\section{A. Impulse response}

The impulse response was predicted to allow the sound reflected from the structures to be heard. Figure 8 shows the impulse response of the scattered pressure reflected from the surface. It is shown for two angles, $0^{\circ}$ and $54^{\circ}$. This was generated using the BEM by predicting the scattered pressure from 0 to $10 \mathrm{kHz}$ at $31.3 \mathrm{~Hz}$ intervals and then applying an inverse Fourier transform. Before applying the transform, a first order low pass Butterworth filter was applied with a $-3 \mathrm{~dB}$ point at $5 \mathrm{kHz}$ to reduce artifacts caused by truncating the spectra appearing in the impulse response.

For normal incidence/reflection, a series of reflections can be seen, spaced apart by about $0.5 \mathrm{~ms}$, which is the time taken for sound to travel from one perforated sheet to a neighboring sheet, and back again. As the angle increases the reflections get weaker. Also, the reflections get closer together as would be expected from simple consideration of geometry.

Ironically, while optical iridescence produces visual beauty, the aural equivalent is not generated by these sound structures. The regularly spaced reflections in the impulse
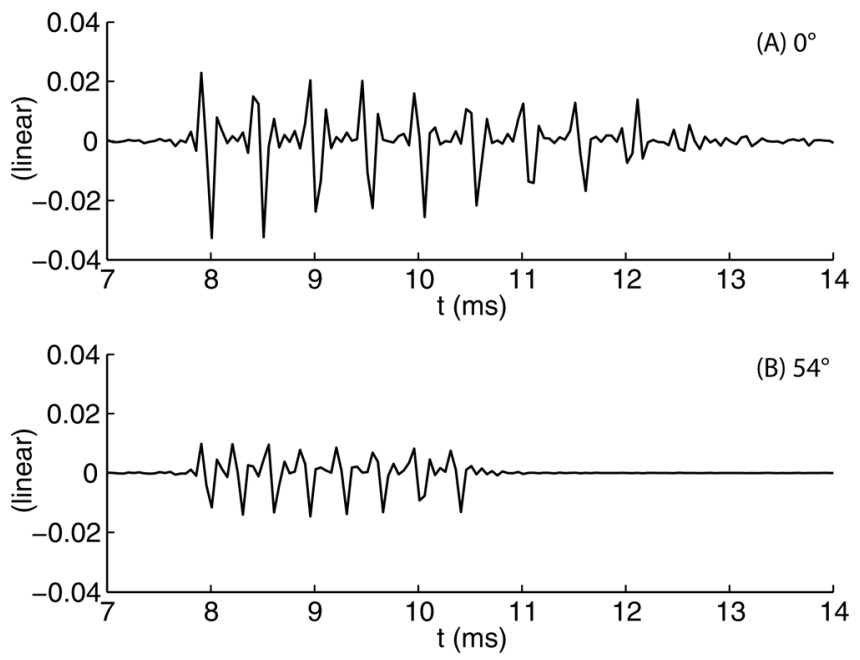

FIG. 8. Impulse responses for an iridescent structure. Geometry same as for Fig. 7. BEM predictions. Shown for two angles of observation: (A) $0^{\circ}$ and (B) $54^{\circ}$. 
responses results in comb filtering, somewhat reminiscent of old-fashion artificial reverberation systems, and the pressure scattered from the surface sounds very colored.

\section{METAMATERIAL}

It is possible to construct a closer analogy to the optical system, one where there are two distinct layers of materials which are roughly quarter of a wavelength in thickness. Figure 9 shows such a construction consisting of three air layers and four layers of a metamaterial. Popa and Cummer ${ }^{16} \mathrm{dem}-$ onstrated how to construct a broadband acoustic metamaterial using periodic arrangements of elements and inclusions which are considerably less than a wavelength in size. They constructed an anisotropic flat film which could bend propagating sound. A preliminary investigation was carried out to see if such an approach could be used to design a material for use in acoustic iridescence.

A slightly simpler construction to that used by Popa and Cummer was adopted. This was done to reduce the number of elements required in the BEM mesh and so decrease the time required for the predictions. A pragmatic trial-and-error approach was taken to determining the geometry shown in Fig. 9. The size of the L-shapes and the gaps between them were taken from the previously-used perforated sheets. As the base shape is an L and anisotropic, these were randomly rotated to make the bulk-properties of the material more isotropic.

Next it was necessary to determine the thickness of the layers necessary to produce iridescence starting at a particular design frequency for normal reflection. One approach would have been to determine the bulk acoustic material properties of the metamaterial and use that in the design work. However, it is also possible to take a more empirical approach. The thickness of the metamaterial was specified $(94.3 \mathrm{~mm})$ and then the scattering from a single layer examined to determine an appropriate design frequency for the iridescence (the frequency of the first harmonic for normal incidence and reflection). To do this, the energy scattered from a single layer of the metamaterial was predicted for normal incidence and reflection to determine the frequency at which maximum reflected energy occurred. The lowest frequency at which this happened was $650 \mathrm{~Hz}$ (which is when the wavelength in air is 5.5 times the thickness of the

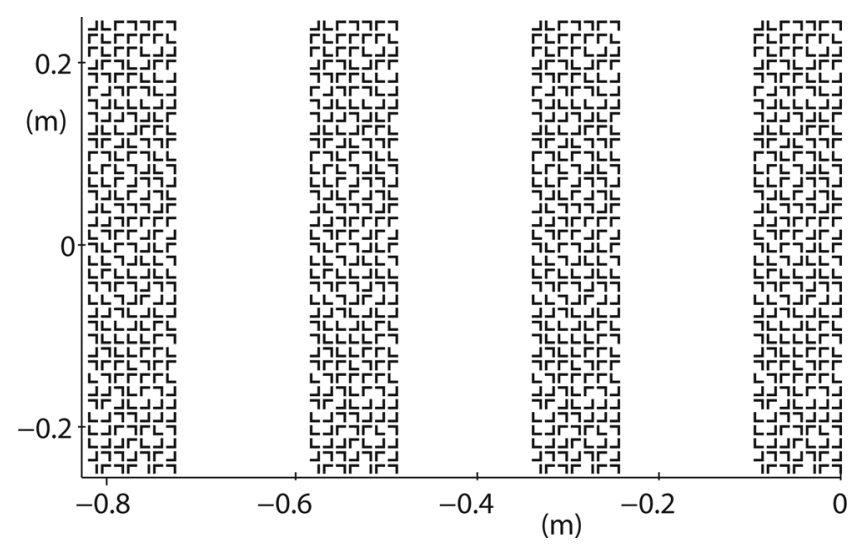

FIG. 9. Construction for alternative structure.

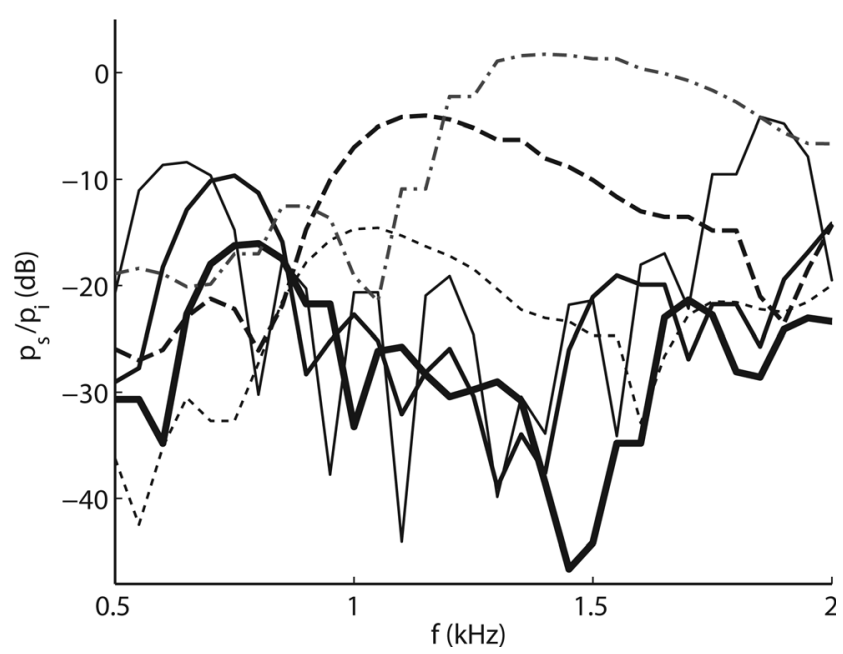

FIG. 10. The scattered pressure level spectrum for the iridescent metamaterial construction shown in Fig. 9. Various angles of incidence/reflection

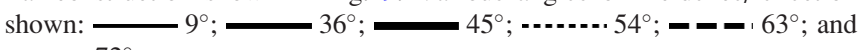
$-72^{\circ}$

metamaterial). The thicknesses of the air layers were then set to be a quarter of the wavelength in air at $650 \mathrm{~Hz}$.

Figure 10 shows the scattered pressure level for the construction shown in Fig. 9. The graph shows various incident/ reflection angles. As the angle gets larger, the peak of the first harmonic increases in frequency demonstrating the desired iridescence. However, the variation in the peak energy is different to the previous construction using just perforated sheets. The peak energy decreases between $0^{\circ}$ and about $50^{\circ}$, but then increases as the angle of observation gets larger. Further exploration of the metamaterial properties is needed to determine why this change in level occurs.

\section{CONCLUSIONS}

Structures which create iridescence with sound waves have been predicted and measured. While inspired by natural optical iridescent structures, alterations are necessary to achieve the necessary backscattered reflections which constructively interfere. The simplest construction achieved this using perforated sheets spaced by half a wavelength. Measurements of two such structures were made, and the results shown to match predictions using a BEM. A more simple prediction model which exploited transfer matrix modeling and the Kirchhoff boundary condition was less accurate but was useful in early design work. Measurements and predictions show the frequencies which are strongly reflecting increasing as the observation angle gets larger, thus mimicking optical iridescence seen in nature. A brief investigation showed that an acoustic metamaterial could also generate iridescence.

\section{ACKNOWLEDGMENTS}

Peter Vukusic kindly provided valuable advice on equivalent optical systems and provided Fig. 1. Jon Hargreaves and Rick Hughes carried out the measurements of the constructions.

${ }^{1}$ The Oxford Dictionary of English (Revised Edition) edited by Catherine Soanes and Angus Stevenson, iridescent adjective, (Oxford University Press, 2005) 
${ }^{2}$ P. Vukusic, "Materials science. Evolutionary photonics with a twist," Science 325, 398-399 (2009).

${ }^{3}$ P. Vukusic, J. R. Sambles, C. R. Lawrence, and R. J. Wootton, “Quantified interference and diffraction in single Morpho butterfly scales," Proc. R. Soc. London, Ser. B 266, 1403-1411 (1999).

${ }^{4} \mathrm{~S}$. Berthier, "Determination of the cuticle index of the scales of the iridescent butterfly Morpho menelaus," Opt. Commun. 228, 349-356 (2003).

${ }^{5}$ T. Miyashita, "Sonic crystals and sonic wave-guides," Meas. Sci. Technol. 16(5), 47-63 (2005).

${ }^{6}$ L.-Y. Wu and L.-W. Chen, "The dispersion characteristics of sonic crystals consisting of elliptic cylinders," J. Phys. D: Appl. Phys. 40, 7579-7583 (2007).

${ }^{7}$ A. J. Burton, “The solution of Helmholtz' equation in exterior domains using integral equations," Report No. NAC30, National Physical Laboratory (1973).

${ }^{8}$ T. Terai, "On calculation of sound fields around three dimensional objects by integral equation methods," J. Sound Vib. 69, 71-100 (1980).

${ }^{9}$ T. J. Cox and P. D'Antonio, Acoustic Absorbers and Diffusers: Theory, Design and Application (Taylor \& Francis, London, 2009), p. 218.
${ }^{10}$ L. Cremer and H. A. Müller, Principles and Applications of Room Acoustics, translated by T. J. Shultz (Applied Science, London, 1982), Vol. 2, p. 187.

${ }^{11}$ T. J. Cox and P. D'Antonio, Acoustic Absorbers and Diffusers: Theory, Design and Application (Taylor \& Francis, London, 2009), p. 24.

${ }^{12}$ T. J. Cox and P. D'Antonio, Acoustic Absorbers and Diffusers: Theory, Design and Application (Taylor \& Francis, London, 2009), pp. 268-269.

${ }^{13}$ T. J. Cox and P. D'Antonio, Acoustic Absorbers and Diffusers: Theory, Design and Application (Taylor \& Francis, London, 2009), pp. 273-274.

${ }^{14}$ J. F. Allard, W. Lauriks, and C. Verhaegen, "The acoustic sound field above a porous layer and the estimation of the acoustic surface impedance from free-field measurements," J. Acoust. Soc. Am. 91(5), 3057-3060 (1992).

${ }^{15}$ AES-4id-2001 information document for room acoustics and sound reinforcement systems-characterization and measurement of surface scattering uniformity, Audio Engineering Society, (2001).

${ }^{16} \mathrm{~B}$. Popa and S. A. Cummer, "Design and characterization of broadband acoustic composite metamaterials," Phys. Rev. B 80, 174303 (2009). 